
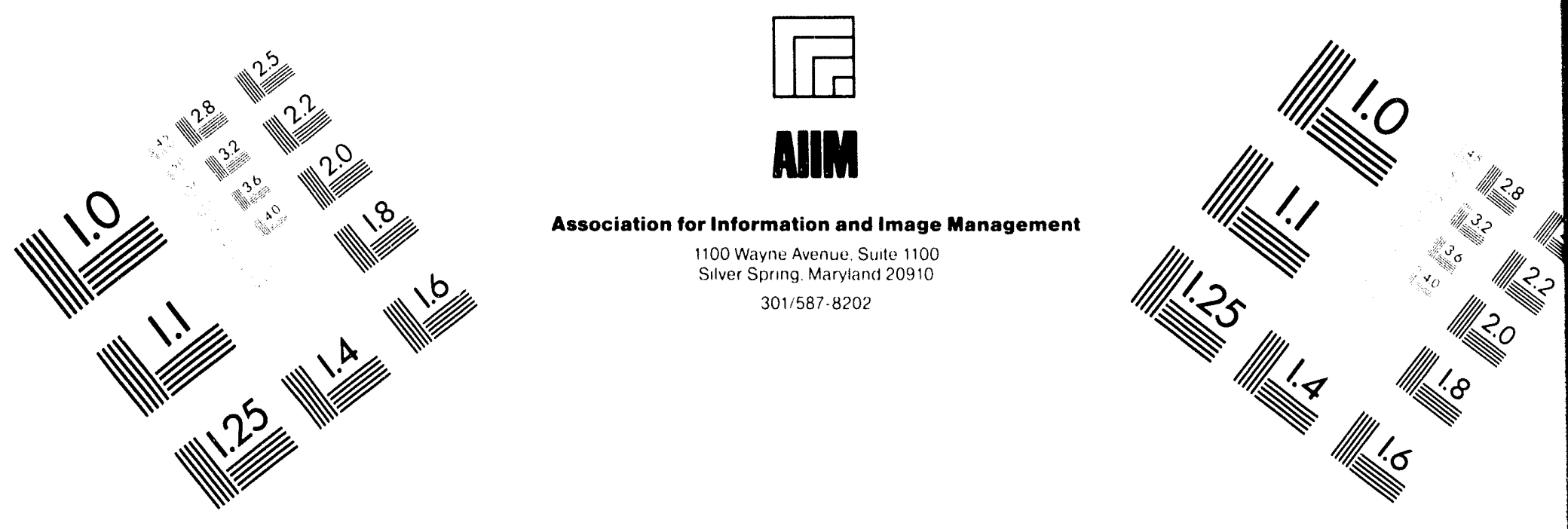

\title{
Centimeter
}

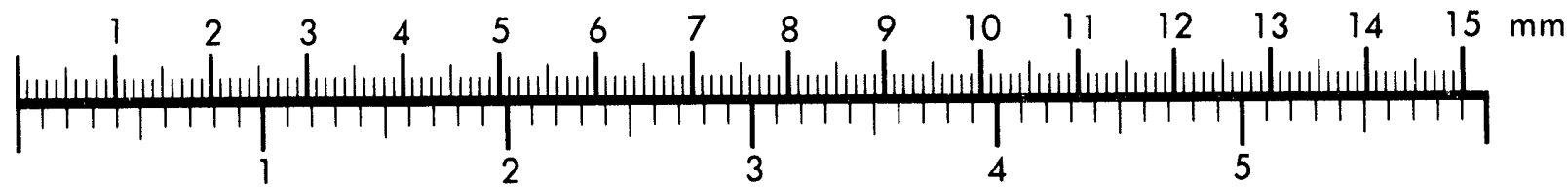

Inches
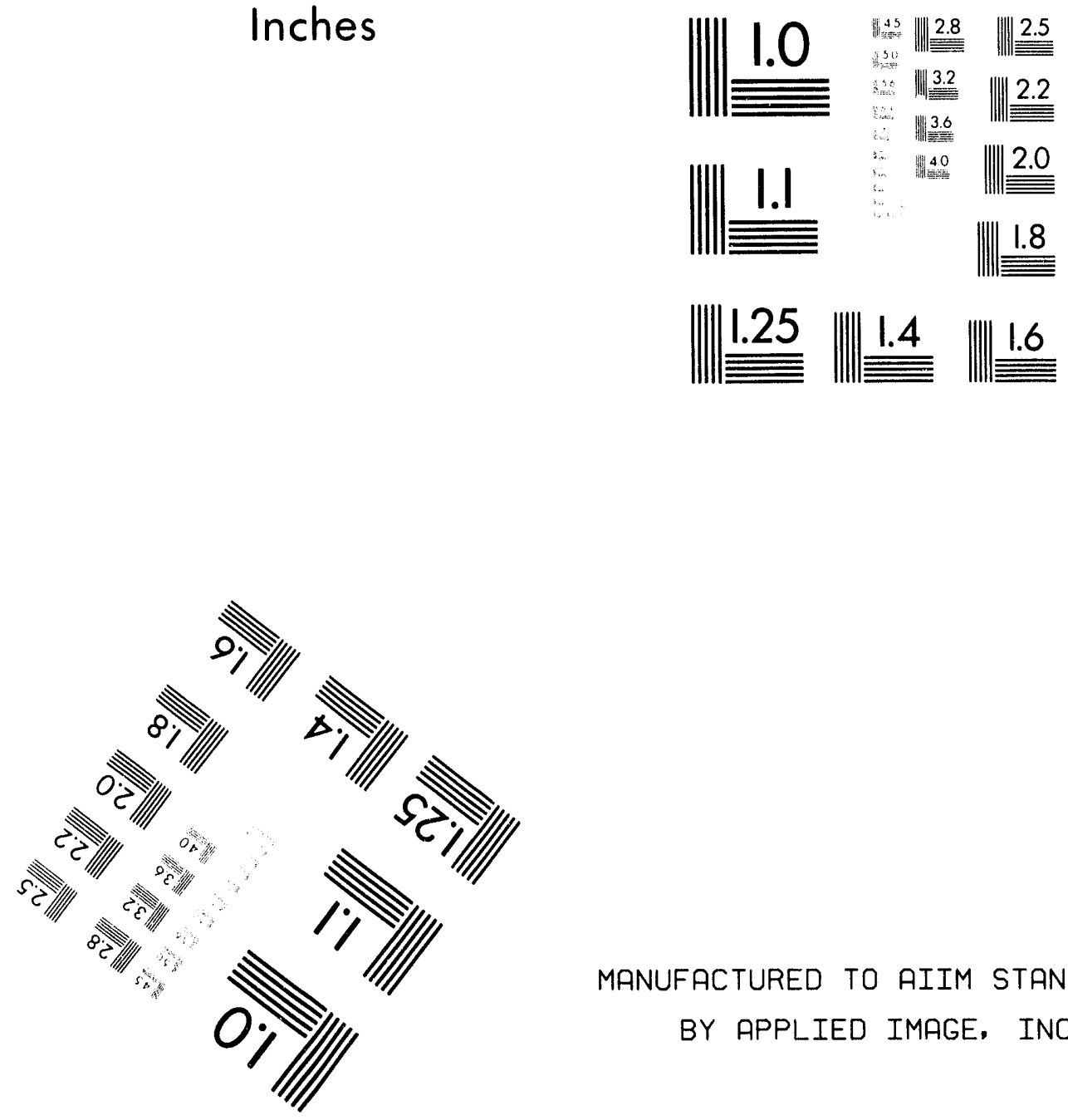

MANUFACTURED TO AIIM STANDARDS

BY APPLIED IMAGE, INC.

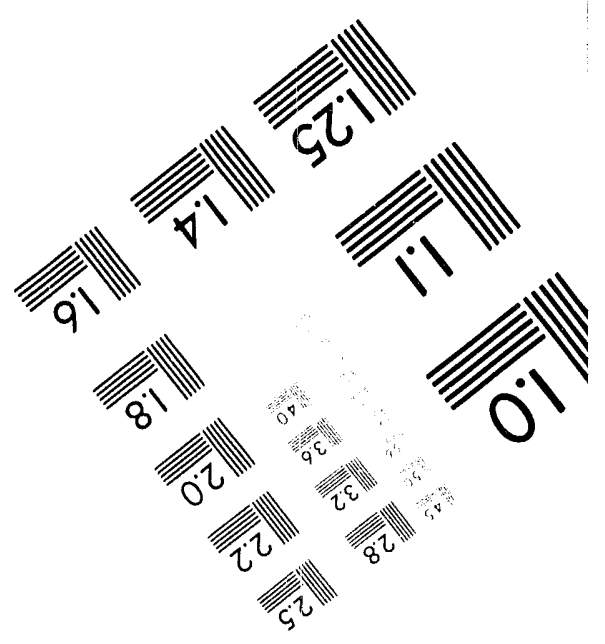



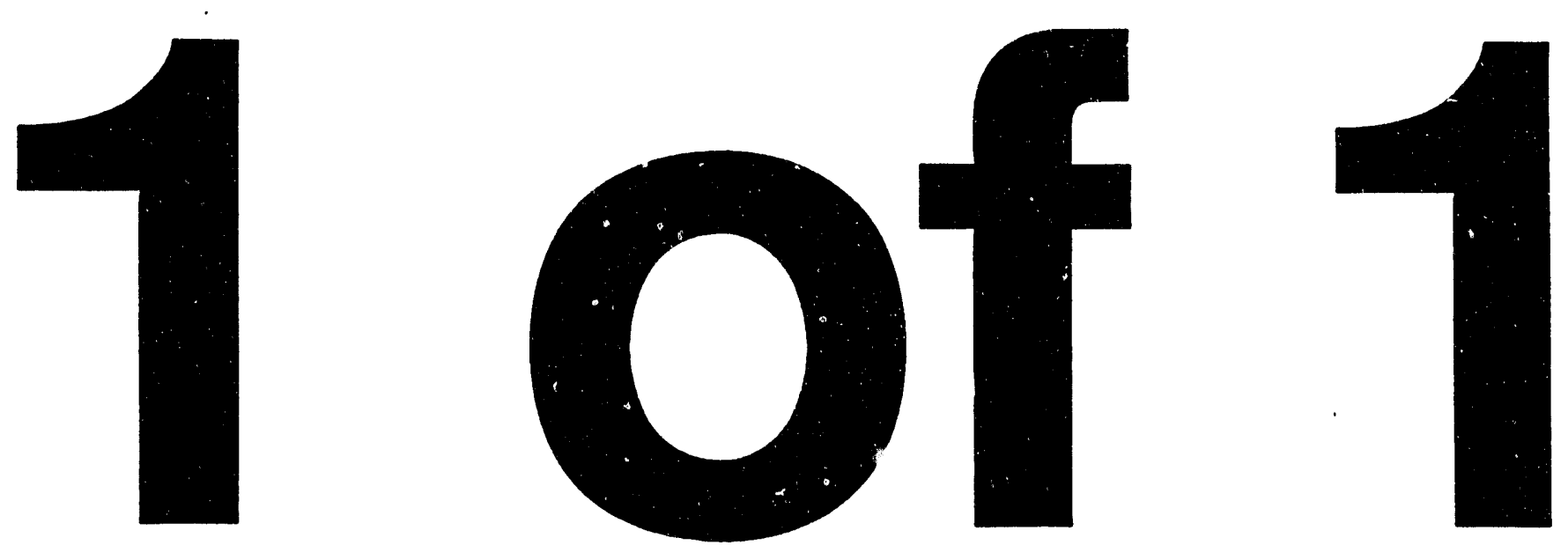
BW -64444

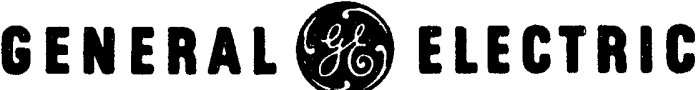

HANFORD ATOMIC PRODUCTB OPERATION - RICHLAND, WABHINGTON
SERIES AND COPY NO.

DATE

March 28, 1960

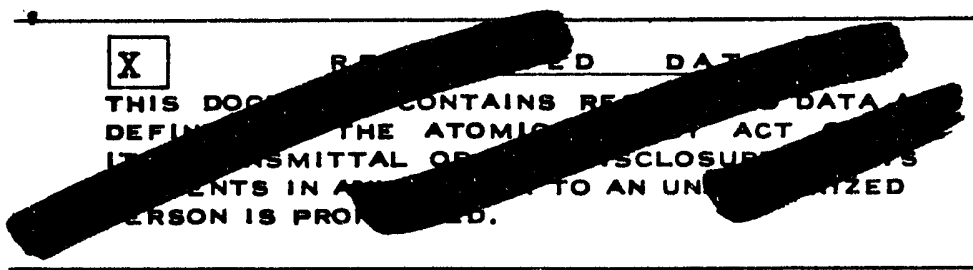

OTHER OFFICIAL CLASBIFIED INFORMATION

THIS MATERIAL CONTAINS INFORMATION AFFECTING THE NATIONAL DEFENSE OF THE UNITED STATES WITHIN THE MEANING OF the EsPIONAGE LAWS, TITLE I8, U.S.C., SECS. TO3 AND 794, THE TRANSMISSION OR REVELATION OF WHICH IN ANY MANNER TO AN UNAUTHORIZED PERSON IS PROHIBITED BY LAW.
TITLE

\section{RRSUNG OF KER LOOP IRRADIATION TESTS FOR 1957, 1958, AND 1959}

\section{AUTHOR}

W. K. KRAIZIRR

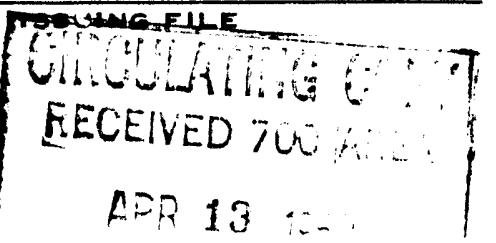

RETIITN TO

THIS DOCUMENT MUST SEFT UNATTENDED TO IT. WHEN NOT

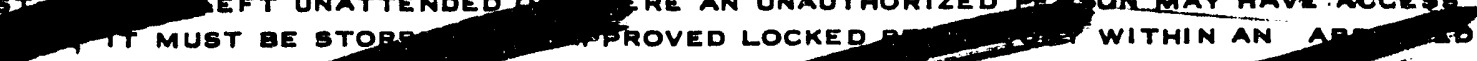

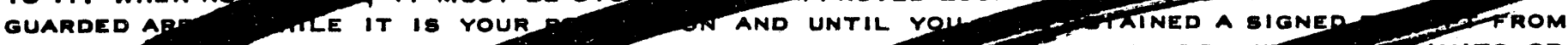

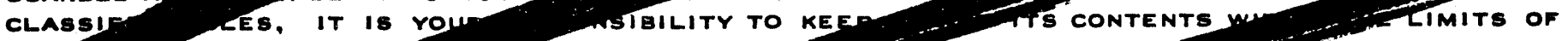

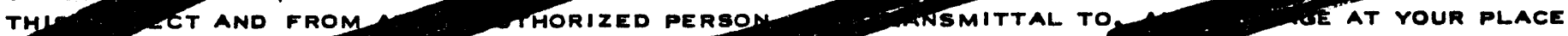

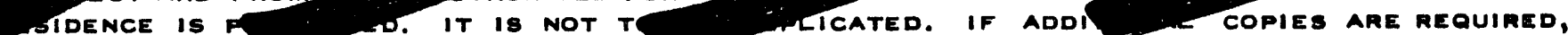
AIN THEM FROM IUL RELATED ISSUING FIL HLL PERSONS READING THIS DOCUMENT ARE REQUESTED TO SIGN IN THE BPACE PROVIDED BELOW.

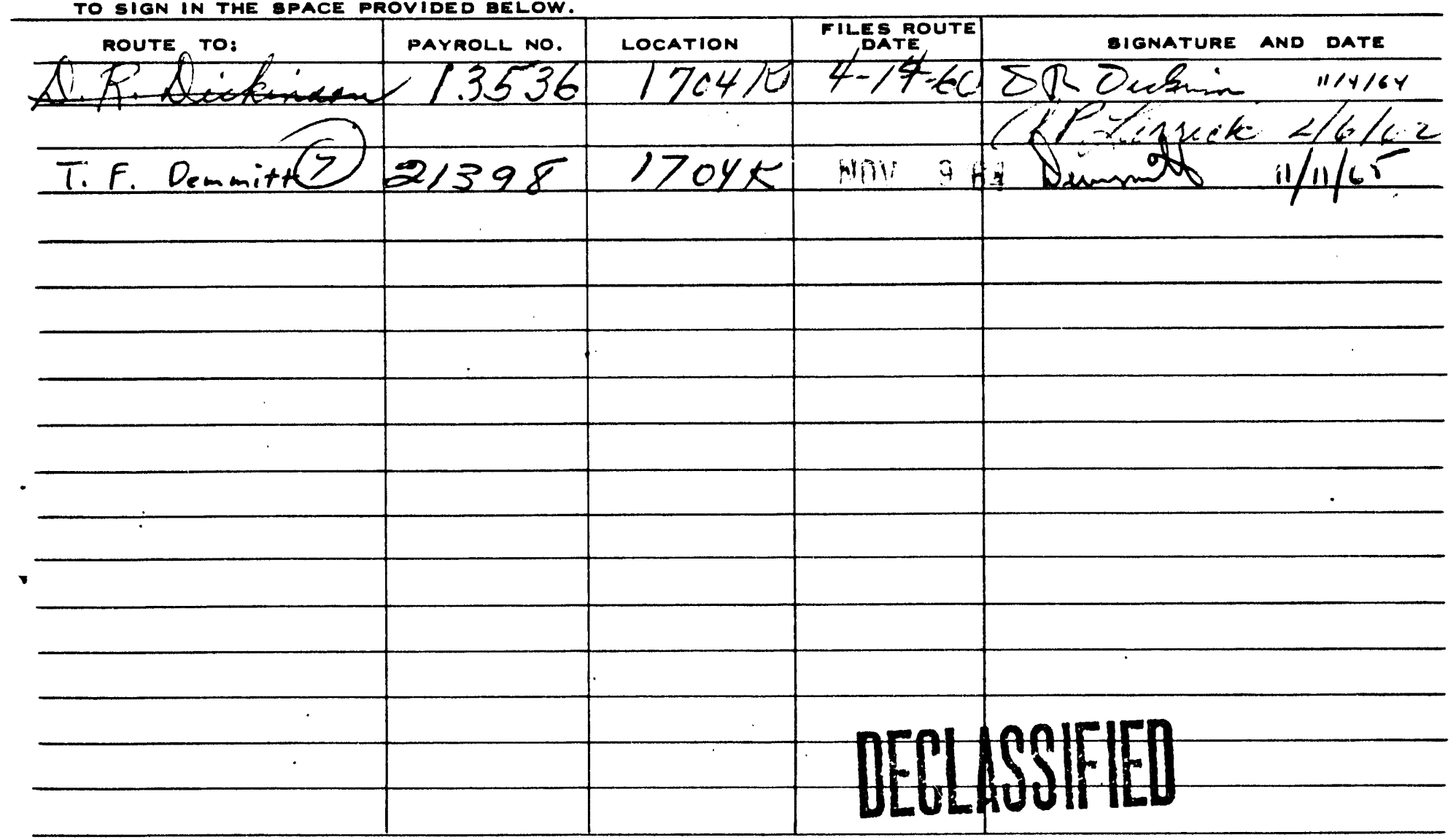

54-3000-340 (3-55) ALC.eE NCELAND. WASH 
RESUME OF KER LOOP IRRADIATION TESTS FOR 1957, 1958, AND 1959

by

\author{
W. K. Kratzer
}

March 20, 1960

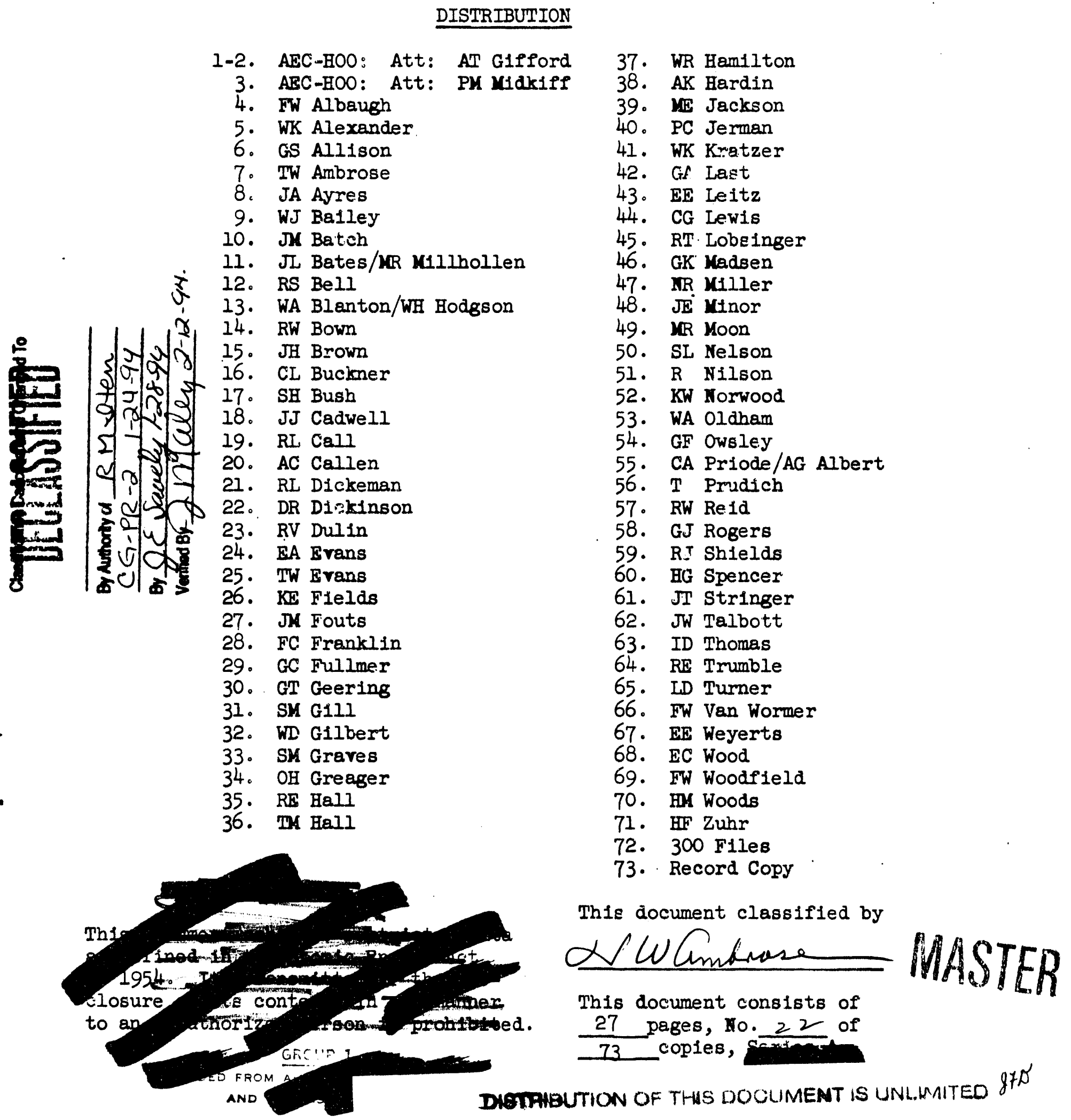


RESULE OF KER LOOP IRRADIATION TESTS FOR 1957, 1958, AND 1959

In 1957 three of the four h1gh temperature recirculating loops in KE Reactor were put into service and in 1958 the fourth loop was started up. The primary purpose of the loops has been high temperature 1rradiation testing of fuel elements in support of the IPR fuel element development program, although tests have been run to provide information for PRTR fuel development and high temperature aluminum corrosion programs. Coolant technology studies relating to activity bulld-up, crud formation, corrosion, fallure detection, and decontamination are carried on in conjunction with the fuel element tests.

The purpose of this document is to provide a resume of all fuel element test Irradiations in the KRR Loops from the time they started up to the end of CY-1959. A description of each test, its operating conditions, a chronological history of each loop, and a bibliography of documents pertaining to the tests are included. Supplements to this report will be issued periodically to bring the information up to date.

\section{Description of the Loops}

The four loops consist of high pressure Z1rcaloy-2 process tubes running frontto-rear in the high flux region of $\mathrm{KF}$ Reactor. The loops contain facilities for controlling the flow, pressure, temperature, and composition of the coolant. Charging and discharging can only take place during reactor outages, and, in general, loop operation is subordinate to the operation of $\mathrm{KB}$ Reactor as a production reactor. Table I lists the main characteristics of the loops. The KER Operations Manual $(1)^{*}$ and Process Standards, Reactor, $(2)$ contain a more complete deseription of the operating procedures and restrictions.

\section{Production Test Design Limitations}

Production test temperatures and operating conditions are limited by Process Standards, based on loop mechanical and hydraulic characteristics, and by the need for loop operating flexibility. Process Standards specify limiting ralues of pressure, temperature, and flow variation. Automatic trips are provided to shut the reactor down if these values are exceeded. Reliable loop operation requires an operating range in which any particular parameter can vary without accidentally exceeding the trip setting. The bottom of this range, therefore, is the maximum operating value arailable for normal testing. For example, the maximum coolant outlet temperature for loop 2 is specifled as $303^{\circ} \mathrm{C}$ by the Process Standards. Loop ope ration requires a $15^{\circ} \mathrm{C}$ spread between the operating temperature and the trip temperature, giving a $288^{\circ} \mathrm{C}$ maximum outlet temperature available for normal operation.

Most of the fuel element production test 1rradiations in the loops are designed to provide the maximum fuel element temperature arailable within the limitations of the following design parameters:

a. The outlet temperature of the process tube coolant shall not exceed a temperature $25^{\circ} \mathrm{C}$ less than the saturation temperature of the system.

* Superscript numbers refer to reports or documents listed in the bibliography. 


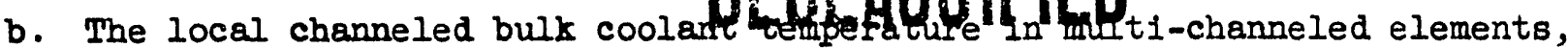
such as the tubular and cluster elements, shall not exceed the saturation temperature of the system either during normal operation or at the limitIng trip conditions - high outlet temperature trip, low system pressure trip, and low coolant flow trip.

c. The fuel element surface temperature should not exceed the system saturat,ion temperature during normal operation unless surface bolling is spec1f1cally desired.

d. Subcooled burnout should not occur during the simultaneous approach to the high outlet temperature trip, low flow trip, and low pressure trip.

e. Although not mandatory, it is desirable to maintain the tube power low enough to permit reduction of the outlet temperature to $100{ }^{\circ} \mathrm{C}$ so that the loop can be switched from recirculation to single pass operation without shutting the reactor down in the event of a leak or some other occurrence that would prevent continued high pressure operation. Maximum tube powers at which this can be accomplished are about $675 \mathrm{KW}$ for $100 \mathrm{p} 1$ and $800 \mathrm{KW}$ in loops 2,3 , and 4 .

\section{Description of the Tables and Figures}

Table I provides a list of the principal loop characteristics that most directly influence fuel element test conditions. Table 2 gives a description of the main fuel elements that hare been tested. Table 3 provides the purpose, test conditions, duration of irradiation, exposure, and results of each test. In general, power, exposure, and surface temperature values apply only to the principal fuel elements in the test. Powers and temperatures are normal equilibrium values which best categorize the operating conditions. Average ralues will be lower because of non-equilibrium and low temperature operation. Periods of low temperature operation for more than $30 \%$ of the irradiation time hare been noted. In cases of heterogeneous loadings, power values are at best approximate since there 18 no way of experimentally measuring the power of the different elements in the charge. Ho one number can categorize tubular element surface temperatures, since there are three flow channels with asymmetrical flow and heat distributions and four heat generating surfeces with different heat fluxes across each surface. The number given is the highest surface temperature predicted for the charge, and temperatures at other surfaces and other positions in the charge should be lower.

At best, the numbers given in Table 3 provide a means of assessing relative test severity. Interpretation of data must be based on a detailed analysis of each test, which is beyond the scope of this report.

Figure 1 gires a chronological listing of the tests run in each loop and a graphical representation of $100 p$ and reactor operating periods.

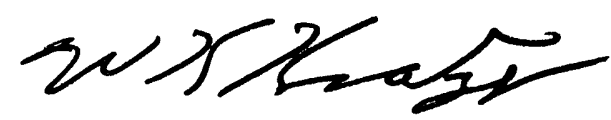

Reactor Fuels Unit

Process \& Reactor Development Subsection Research and Fnglneering Section IRRADIATION PROCESSING DEPARTMENT 
EW-64444

Page 4

TABIE I

KER LOOP CEARACTERISTICS, DECEYBER, 1959

\begin{tabular}{|c|c|c|c|c|c|c|c|c|c|c|c|}
\hline \multirow{3}{*}{ LOOP } & \multicolumn{4}{|c|}{ PROCESS TURE } & \multirow{3}{*}{$\begin{array}{l}\text { OUT OF PIIE } \\
\text { LOOP NAT'L. }\end{array}$} & \multicolumn{2}{|c|}{ SYSTEY PRESSURE, PSIG } & \multicolumn{2}{|c|}{ COOLANT FLOW, .GPM } & \multirow{3}{*}{$\begin{array}{l}\text { KAXTWUX OPERATING } \\
\text { COOLANT OUTIET } \\
\text { TEYPERATURE, }{ }^{\circ} \mathrm{C}\end{array}$} & \multirow{3}{*}{$\begin{array}{l}\text { BEATT EXCEANGER } \\
\text { CAPACIIY, KW }\end{array}$} \\
\hline & \multirow[t]{2}{*}{ MAF'L. } & \multirow[t]{2}{*}{ OD II. } & \multicolumn{2}{|c|}{ II II. } & & OPERATIRG & MTNTIM & OPKRATIMG & Fring & & \\
\hline & & & BYDRAULIC & $\begin{array}{l}\text { PLUG } \\
\text { GAUGE }\end{array}$ & & & & & & & \\
\hline$\frac{1}{2160 \mathrm{~KB}}$ & $\mathrm{Zr}-2$ & 2.40 & 2.089 & 2.079 & $\begin{array}{l}\text { Carbon } \\
\text { Steel }\end{array}$ & 1300 & 1250 & 50 & 40 & 270 & 1200 \\
\hline $2864 \mathrm{KR}$ & $\mathrm{Zr}-2$ & 2.40 & 2.030 & 2.023 & $\begin{array}{l}\text { Stainless } \\
\text { Steel }\end{array}$ & 1600 & 1500 & 60 & 48 & 288 & 1200 \\
\hline $\begin{array}{l}3 \\
3565 \mathrm{KR}\end{array}$ & $\mathrm{Zr}-2$ & 2.40 & 2.030 & 2.018 & $\begin{array}{l}\text { Stalnless } \\
\text { Steel }\end{array}$ & 1600 & 1500 & 60 . & 48 & 288 & 1200 \\
\hline $\begin{array}{l}4 \\
4268 \mathrm{KR}\end{array}$ & $2 r-2$ & 2.40 & 2.030 & 2.023 & $\begin{array}{l}\text { Stainless } \\
\text { Steel }\end{array}$ & 1600 & 1500 & 60 & 48 & 288 & 1200 \\
\hline & & & & CHARAC & $\begin{array}{l}\text { UERISTICS PRIOF } \\
3 \text { ON MARCH } 24\end{array}$ & TO FROGESS & $\begin{array}{l}\text { TURE ESPLAd } \\
00 \mathrm{P} 4 \text { OI } \pi\end{array}$ & 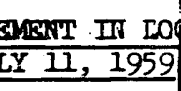 & & & \\
\hline 1 & $\mathrm{Zr}-2$ & 2.40 & 2.089 & 2.079 & $\begin{array}{l}\text { Carbon } \\
\text { Steel }\end{array}$ & 1300 & 1250 & 50 & 40 & 270 & 1200 \\
\hline 2 & $\mathrm{Zr}-2$ & 2.40 & 2.10 & $-\ldots$ & $\begin{array}{l}\text { Stainless } \\
\text { Steel }\end{array}$ & 835 & 785 & 60 & 48 & 245 & 1200 \\
\hline 3 & $\mathrm{Zr}-2$ & 2.40 & 2.10 & $\ldots$ & $\begin{array}{l}\text { Stainless } \\
\text { Steel }\end{array}$ & 835 & 785 & 60 & 48 & 245 & 1200 \\
\hline 4 & $\mathrm{Zr}-\hat{c}$ & 2.40 & 2.10 & $-\cdots$ & $\begin{array}{l}\text { Stainless } \\
\text { Steel }\end{array}$ & 835 & 785 & 60 & 48 & 245 & 1200 \\
\hline
\end{tabular}




\section{UECLSSPFFED}

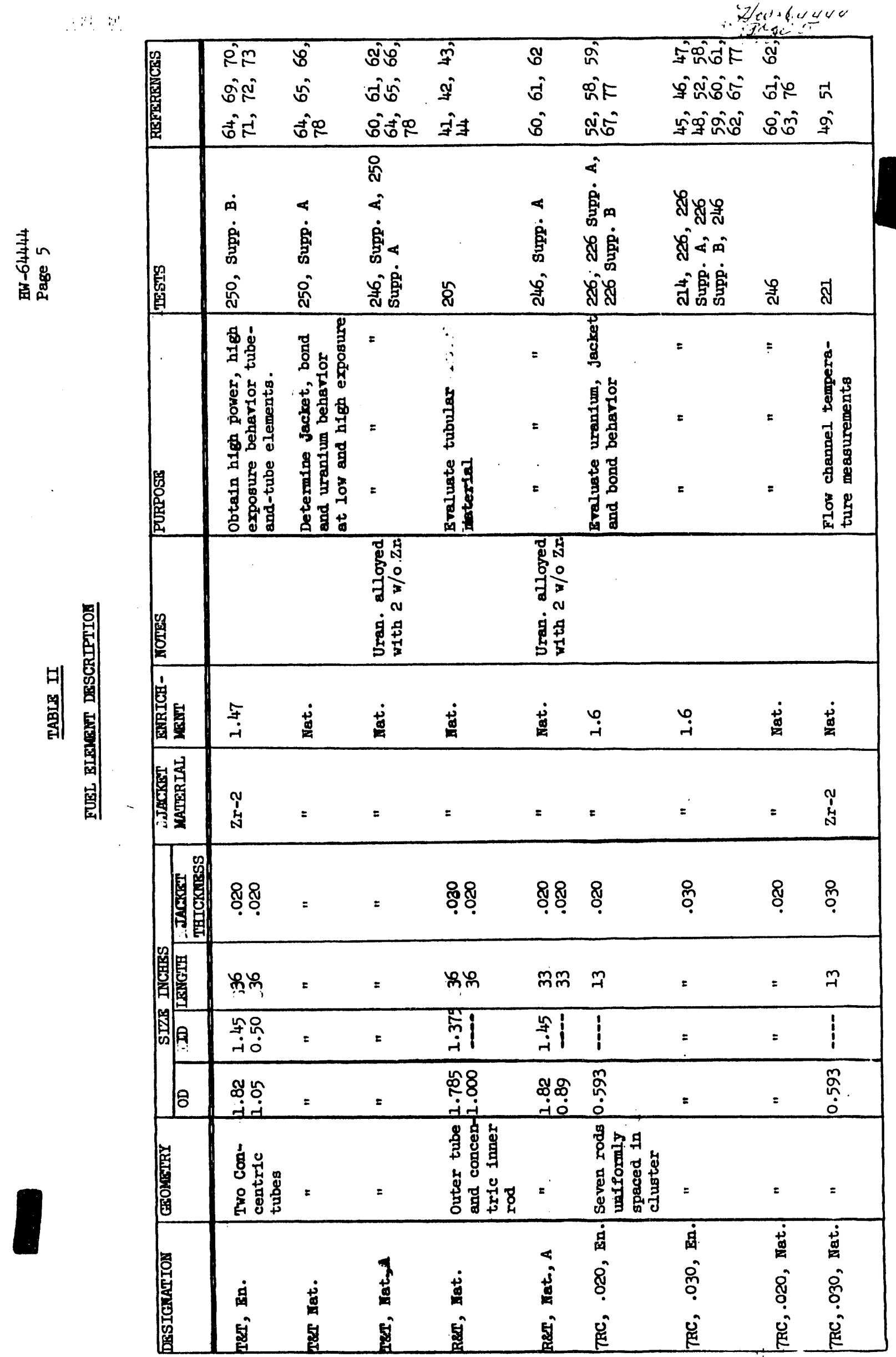



TABLB II (Cont'd)

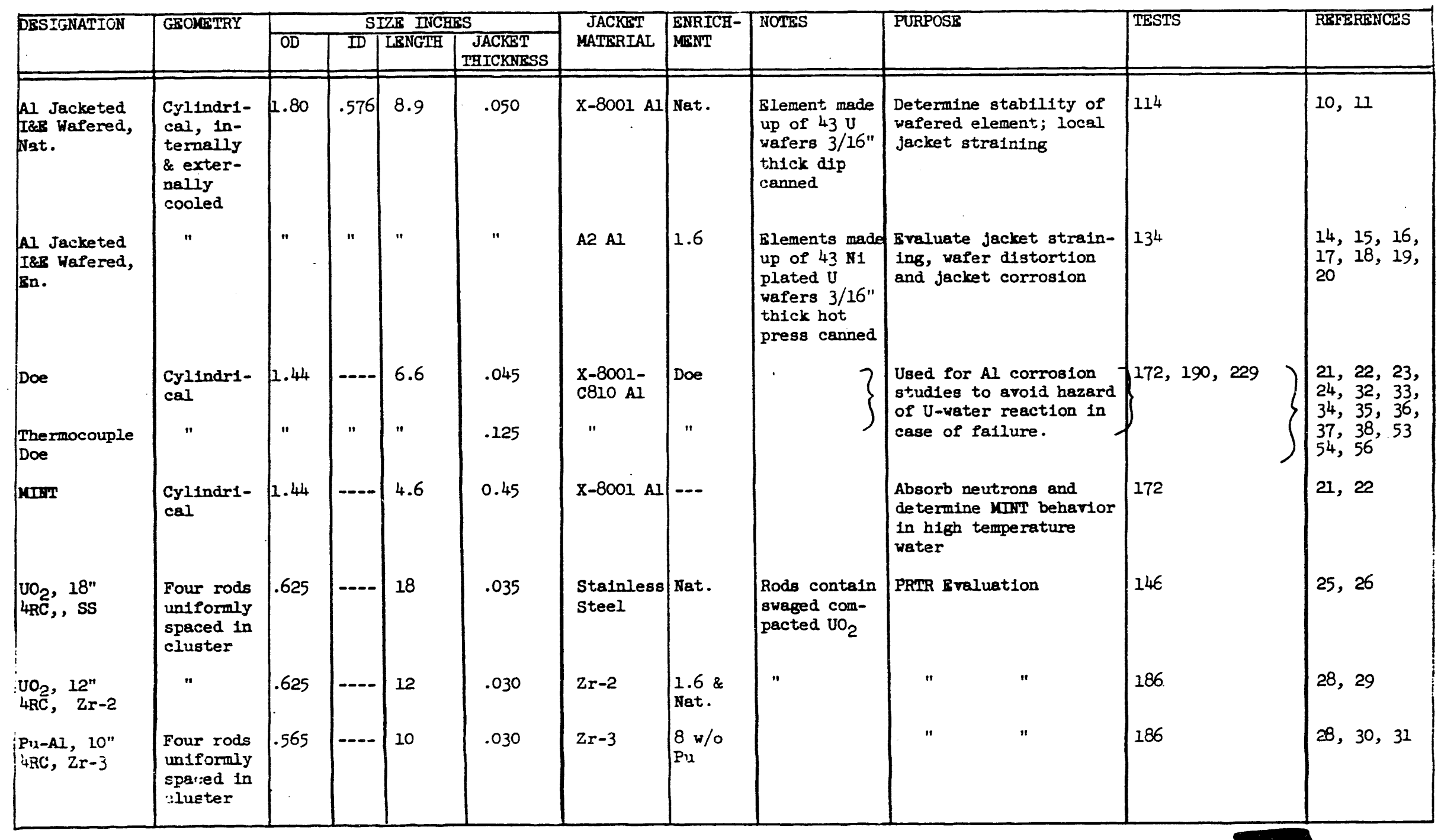




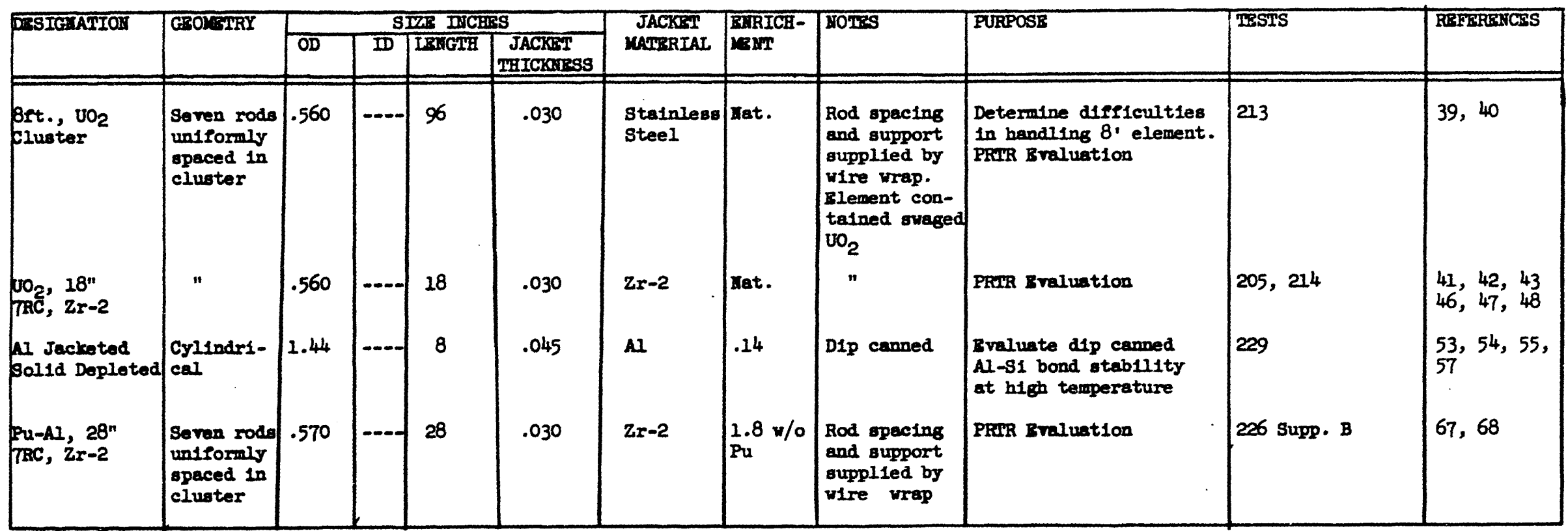



TABLR III (Cont'd)

BN-64444

Page 10

\begin{tabular}{|c|c|c|c|c|c|c|c|c|c|c|c|c|c|}
\hline $\begin{array}{l}\text { PRODUCTION } \\
\text { TEST }\end{array}$ & $\begin{array}{l}\text { CHARGR \& DIS- } \\
\text { CHARGR DAUIS }\end{array}$ & IOOP & \begin{tabular}{|l|} 
ORDIIAL \\
TUSBRR \\
OF LOADIIG \\
II LOOP \\
\end{tabular} & 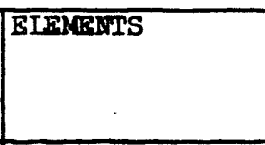 & $\begin{array}{l}\text { POWER } \\
\text { KN/FWT/ } \\
\text { RLENRBNT }\end{array}$ & $\begin{array}{l}\text { HEAT FLUX } \\
\mathrm{B} / \mathrm{HR} \text {. -FT2 }\end{array}$ & $\begin{array}{l}\text { PEMPERA } \\
\text { PUTLET }\end{array}$ & $\begin{array}{l}\text { TURE, O9 } \\
\text { SURFACG }\end{array}$ & COOLART & \begin{tabular}{|l|} 
EXPOS \\
GOAL \\
\end{tabular} & 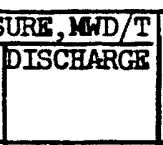 & PURPOSE AND RESULIS & REFGRENCES \\
\hline $144 \mathrm{~A}$ & $\begin{array}{l}12 \text { Feb } 58 \\
15 \text { May } 58\end{array}$ & 1 & 1 & 19 4RC, SS, Nat & 36 & $.19 \times 10^{6}$ & 260 & 280 & pH8， L1OH & 1000 & 580 & $\begin{array}{l}\text { Check inftial loop } \\
\text { operation. Test was } \\
\text { discharged when this } \\
\text { was accomplished }\end{array}$ & 12,13 \\
\hline $134 \mathrm{~A}$ (1) & 17 Aprt1 58 & 4 & 4 & $\begin{array}{l}10 \text { Al jacketed } \\
\text { I\&E wafered, En. }\end{array}$ & 150 & $1.1 \times 10^{6}$ & 240 & 250 & $\begin{array}{l}\mathrm{pH} 4.5, \therefore \\
\mathrm{H}_{3} \mathrm{PO}_{4}\end{array}$ & $\begin{array}{l}\text { pased } \\
\text { pn er } \\
\text { fosion }\end{array}$ & 400 & $\begin{array}{l}\text { Fraluate facket strain } \\
\text { wafer distortion, bond } \\
\text { retention, and jacket } \\
\text { corrosion. Jacket, } \\
\text { wafer and bond un- } \\
\text { affected. Jacket } \\
\text { corrosion less than } \\
\text { antlcipated. }\end{array}$ & $\begin{array}{l}14,15 \\
16,17 \\
18,19 \\
20\end{array}$ \\
\hline $172 A$ & $\begin{array}{l}28 \text { June } 58 \\
9 \text { Hor } 58\end{array}$ & 2 & 5 & $\begin{array}{l}4 \text { 4RC, .030 } \\
\text { En; } 26 \text { Doe; } \\
1 \text { thermocouple } \\
\text { Doe; } 8 \text { somit }\end{array}$ & 90 & $.47 \times 10^{6}$ & 200 & 250 & $\begin{array}{l}\mathrm{pH} 4.5, \mathrm{II} \\
\mathrm{B}_{3} \mathrm{PO}_{4}\end{array}$ & 2000 & 2200 & $\begin{array}{l}\text { Determine Irradiation } \\
\text { beharlor of coextruded } \\
\text { Zr-2 jacketed rod. } \\
\text { Rods increased 3\% in } \\
\text { rolume and hed macro- } \\
\text { cracles in uranium. Ho } \\
\text { gross distortion, no } \\
\text { bond deterioration. } \\
\text { Heglibible corrosion } \\
\text { of Does; fatlure ob- } \\
\text { served on one vmir } \\
\text { piece. }\end{array}$ & $\begin{array}{l}21, \\
23,22\end{array}$ \\
\hline $146 \mathrm{~A}$ & $\begin{array}{l}4 \text { Aug } 58 \\
14 \operatorname{Jan} 59\end{array}$ & 1 & 2 & $\begin{array}{l}2 \mathrm{TRC}, .030- \\
.630, \mathrm{Nat}^{\circ} \\
6 \mathrm{UO}_{2} 18^{\prime \prime} 4 \mathrm{RC} \text {, } \\
\text { ss }\end{array}$ & 70 & $.21 \times 10^{6}$ & 265 & 270 & pHIO, L10H & 1000 & 1250 & $\begin{array}{l}\text { Evaluate seven-rod } \\
\text { cluster geonetry. Ble- } \\
\text { ments showed no dis- } \\
\text { tort1on, no bond deter- } \\
\text { loration, no cracking. } \\
\mathrm{UO}_{2} \text { elements charged } \\
\text { to provide PRIR infor- } \\
\text { mation. }\end{array}$ & $\begin{array}{l}25,26, \\
27\end{array}$ \\
\hline
\end{tabular}




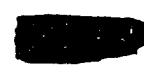

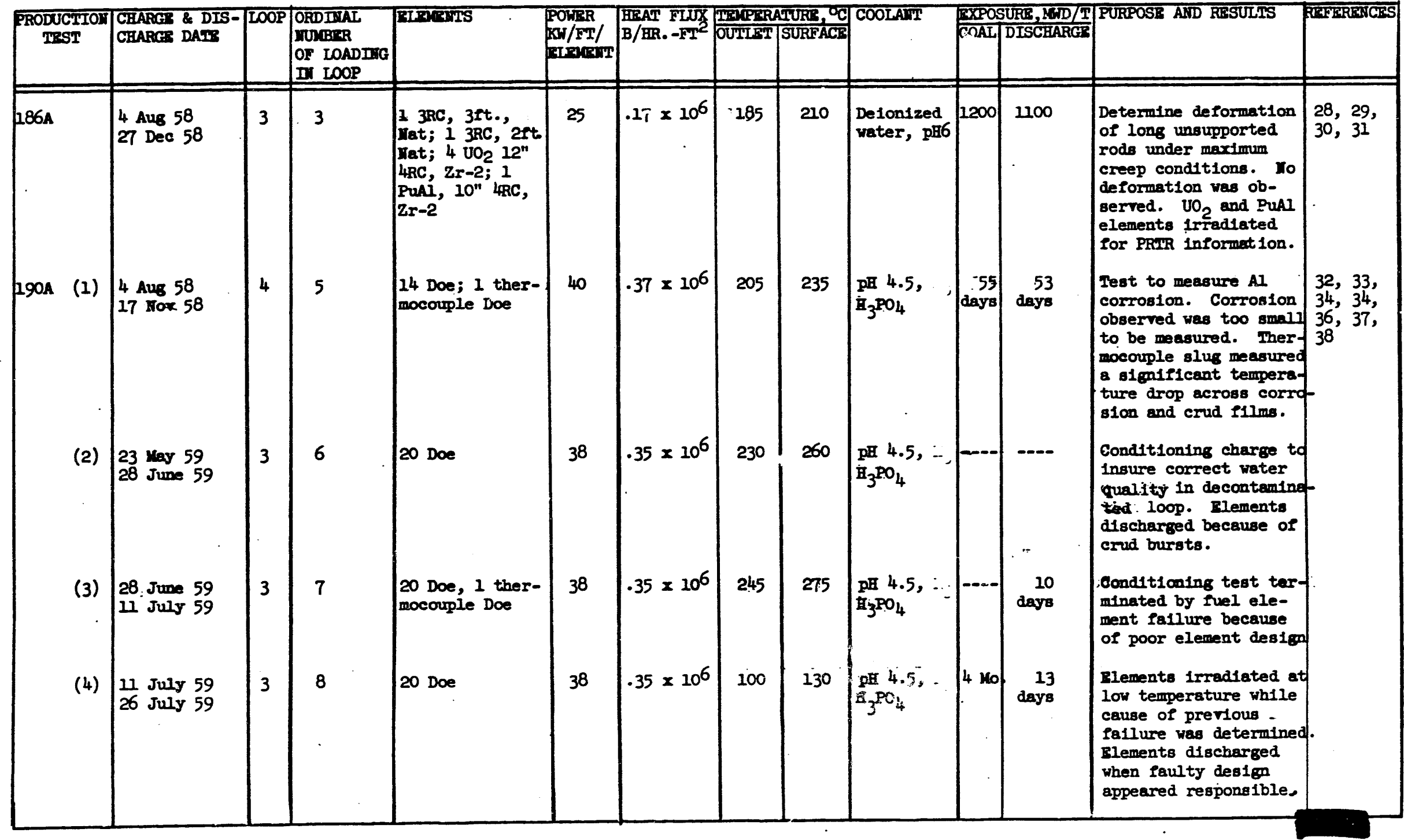




\begin{tabular}{|c|c|c|c|c|c|c|c|c|c|c|c|c|c|}
\hline $\begin{array}{l}\text { PRODUCTION } \\
\text { TRST }\end{array}$ & \begin{tabular}{ll|} 
CHARGR \& DIS- \\
CHARGR DAIIS
\end{tabular} & LOP & \begin{tabular}{|l|} 
ORDINAL \\
NUNGIAR \\
OF LOADTIG \\
II LOOP \\
\end{tabular} & 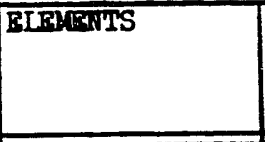 & $\begin{array}{l}\text { POWBR } \\
\text { KW/FT/ } \\
\text { ELBNENT }\end{array}$ & $\begin{array}{l}\text { HEAT FLUX } \\
\text { B/Hr.-FT' } \\
\end{array}$ & $\begin{array}{l}\text { MGFYPERA } \\
\text { DUTTET }\end{array}$ & ATURF, & COOLAWT & COAPOS & SURE, & PURPOSR AND RESULIS & RRFBREWCES \\
\hline $213 A$ & $\begin{array}{l}9 \text { Yor } 58 \\
8 \text { Dec } 58\end{array}$ & 2 & 6 & $\begin{array}{l}18 \mathrm{ft} . \mathrm{UO} 2 \\
\text { cluster }\end{array}$ & 60 & $.20 \times 10^{6}$ & 185 & 260 & pH 1Q, LIOH & $\begin{array}{r}60 \\
\text { days }\end{array}$ & $\begin{array}{r}31 \\
\text { days }\end{array}$ & $\begin{array}{l}\text { Determine difficulty } \\
\text { in charging or irre- } \\
\text { diating long wre- } \\
\text { wrapped cluster. PRIR } \\
\text { application. Ho dif- } \\
\text { ficulty was observed. }\end{array}$ & 39,40 \\
\hline $205 A$ & 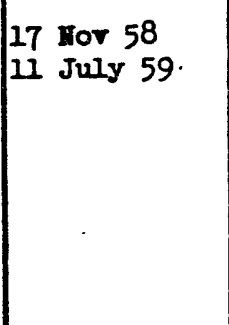 & 4 & 6 & $\begin{array}{l}2 \text { R\&s, Hat; } 1 \\
\mathrm{UO} 2,18^{\prime \prime}, \mathrm{TRC}, \\
\mathrm{Zr}-2\end{array}$ & 73 & $\cdots$ & $\begin{array}{l}220 \\
70 \%\end{array}$ & de time & $\frac{\mathrm{pH} 4.5, \cdots}{\mathrm{H}_{3} \mathrm{PO}_{4}}$ & 2500 & 1900 & $\begin{array}{l}\text { Observe tubular ele- } \\
\text { ment beharlor. Jackiet } \\
\text { corrosion observed as } \\
\text { result of crud f1lm } \\
\text { on surface. One rod } \\
\text { showed } 3 \% \text { rolume in- } \\
\text { crease; one tube was } \\
\text { Internally crecked. } \\
\text { and distorted. }\end{array}$ & $\begin{array}{l}41,42 \\
43,44\end{array}$ \\
\hline 2144 & $\left\{\begin{array}{l}9 \text { Dec } 58 \\
24 \text { March } 59\end{array}\right.$ & 2 & 7 & $\begin{array}{l}4 \mathrm{TRC}, .030 \\
\mathrm{Rn} ; 1 \mathrm{RBC}, 3 \\
\mathrm{ft}, \mathrm{Rn} ; 2 \mathrm{UO}_{3} \\
18 ", \mathrm{TRC}, \mathrm{Zr}-\mathrm{C}\end{array}$ & 140 & $.44 \times 10^{6}$ & 203 & 260 & $\mathrm{pH} 10$, IIOH & 3500 & 1600 & $\begin{array}{l}\text { Test was to dotermine } \\
\text { h1gh power, h1gh ex- } \\
\text { posure beharlor of } \\
\text { materlal. Blements } \\
\text { discharged with fail- } \\
\text { ure attributed to } \\
\text { faulty } \mathrm{Zr}-2 \text { jacket. }\end{array}$ & $\begin{array}{l}45,46 \\
47,48\end{array}$ \\
\hline 2214 & $\begin{array}{l}27 \text { Dec } 58 \\
4 \text { Feb } 59\end{array}$ & 3 & 4 & $\begin{array}{l}8 \mathrm{TRC}, .030 \text {, } \\
\text { Hat. }\end{array}$ & 72 & $.23 \times 10^{6}$ & 200 & 250 & $\begin{array}{l}\text { Delonized } \\
\text { water, pH6 }\end{array}$ & $\begin{array}{l}1 \text { vo } \\
\text { Oper } \\
\text { ation }\end{array}$ & 290 & $\begin{array}{l}\text { Heasure flow channel } \\
\text { temperature. Rat1os } \\
\text { obtained for th1s } \\
\text { fuel and tube size. }\end{array}$ & $\begin{array}{l}49,50, \\
51\end{array}$ \\
\hline 226 A & $\begin{array}{l}14 \text { Jan } 59 \\
29 \text { Karch } 59\end{array}$ & 1 & 3 & $\begin{array}{l}4 \mathrm{TRC}, .020, \mathrm{En} ; \\
3 \mathrm{TRC}, .030, \mathrm{En} .\end{array}$ & 130 & $.41 \times 10^{6}$ & $\begin{array}{l}225 \\
30 \%\end{array}$ & de time & $\mathrm{pH} 10, \mathrm{L1OH}$ & 4500 & 1200 & $\begin{array}{l}\text { Test was to compare } \\
\text {.020 and .030 inch } \\
\text { jacketed elements at } \\
\text { high power and expos- } \\
\text { ure. Test was dis- } \\
\text { sharged when loop } \\
\text { operating difficul- } \\
\text { ties pre rented con- } \\
\text { t,inued operation. }\end{array}$ & 52 \\
\hline
\end{tabular}





\begin{tabular}{|c|c|c|c|c|c|c|c|c|c|c|c|c|c|}
\hline $\begin{array}{l}\text { PRODUCTION } \\
\text { TEST }\end{array}$ & $\begin{array}{l}\text { CHARGS \& DIS- } \\
\text { CHARGI DATS }\end{array}$ & LOOP & \begin{tabular}{|l|} 
RDDINAL \\
NUNGBAR \\
OF LOADING \\
IN LOOP \\
\end{tabular} & ET:FAENTS & \begin{tabular}{l|} 
POWER \\
$\mathrm{KW} / \mathrm{FT} /$ \\
$\mathrm{ELGHWNT}$
\end{tabular} & $\begin{array}{c}\text { HEAT FLUX } \\
\text { B/HR.-FT2 } \\
.\end{array}$ & TEAPERA & $\begin{array}{l}\text { ATURR,OC } \\
\text { SURFACF }\end{array}$ & COOLANT & $\begin{array}{l}\text { EXXOS } \\
\text { COAL }\end{array}$ & $\begin{array}{l}\text { SURE, BWW/T } \\
\text { DISCHARGES }\end{array}$ & PURPOST AND RESULIS & RGFBREACES \\
\hline $\begin{array}{l}\text { 250A } \\
\text { Supp A }\end{array}$ & $\begin{array}{l}28 \text { June } 59 \\
31 \text { July } 59\end{array}$ & 1 & .5 & $\begin{array}{l}2 \text { Ter, Hat; } \\
2 \text { Ter, Hat, A }\end{array}$ & 60 & 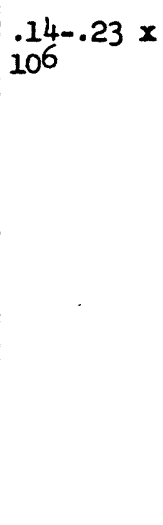 & 270 & $\begin{array}{l}270- \\
290\end{array}$ & . & 2500 & 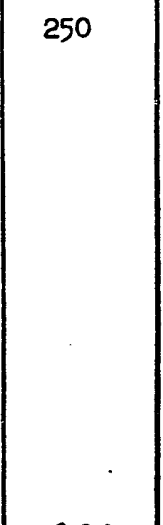 & $\begin{array}{l}\text { Determine facket, } \\
\text { bond, and urantum } \\
\text { beharlor of tubular } \\
\text { fuel elements at high } \\
\text { exposure. Test dis- } \\
\text { charged with fallure. } \\
\text { Location, nature, and } \\
\text { cause of fallure not } \\
\text { determined. Bumplng } \\
\text { observed on racuum } \\
\text { beta heat treated slow } \\
\text { cooled tubes. Radial } \\
\text { cracks obserred on one } \\
\text { tube examined metallo- } \\
\text { graphlcally. }\end{array}$ & $\begin{array}{l}64,65 \\
66,78 \\
\end{array}$ \\
\hline $\begin{array}{l}\text { 226A } \\
\text { Supp B }\end{array}$ & 24 Aug 59 & 1 & 6 & $\begin{array}{l}4 \mathrm{TRC}, .020, \mathrm{Fn} ; \\
2 \mathrm{TRC}, .030, \mathrm{En} ; \\
1 \mathrm{PuAl}, 28^{\prime \prime}, \mathrm{TRC} \text {, } \\
\mathrm{Zr}-2\end{array}$ & 128 & $.40 \times 10^{6}$ & 230 & 310 & pH 1Q, LIOH & 4500 & 2500 & $\begin{array}{l}\text { Compare } 20 \text { and } 30 \\
\text { mII - jackets, deter- } \\
\text { mine bond and uranfum } \\
\text { beharior at h1 eg ex- } \\
\text { posure. Test was } \\
\text { st1ll running at end } \\
\text { of CY-59 and had } \\
\text { attained an exposure } \\
\text { of } 1770 \mathrm{wD} / \mathrm{I} \text {. }\end{array}$ & 67,68 \\
\hline
\end{tabular}


TABLS III (Con'd)

\begin{tabular}{|c|c|c|c|c|c|c|c|c|c|c|c|c|c|}
\hline $\begin{array}{l}\text { PRODUCTION } \\
\text { TEST }\end{array}$ & $\begin{array}{l}\text { CHARGS \& DIS- } \\
\text { GHARGB DATS }\end{array}$ & LOOP & 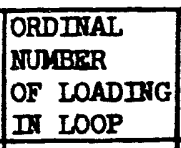 & ELBATENTS & \begin{tabular}{|l|} 
POWER \\
KW/FT/ \\
RLEMEAMT
\end{tabular} & $\begin{array}{l}\mathrm{HRAT} \text { FLUX } \\
\mathrm{B} / \mathrm{HR} /-\mathrm{FT}^{2}\end{array}$ & $\begin{array}{l}\text { THEYPLRA } \\
\text { OUTLET }\end{array}$ & $\begin{array}{l}\text { ATURE, OC } \\
\text { SURFACE }\end{array}$ & COOLANT & EXPOS & $\begin{array}{l}\text { SURB, KAFD/T } \\
\text { DISCEARGE }\end{array}$ & PURPOSE AKD RISSULIS & REFFREAXCES \\
\hline $\begin{array}{l}\text { b50 A } \\
\text { Supp B }\end{array}$ & $\begin{array}{l}7 \text { Sept } 59 \\
30 \text { Sept } 59\end{array}$ & 3 & 9 & 3 T\&T, En. & 92 & 106 & $\begin{array}{l}252 \\
70 \% \text { of } \\
\text { time } \\
283 \\
30 \% \text { of } \\
\text { time }\end{array}$ & $\begin{array}{l}280- \\
310\end{array}$ & $\begin{array}{l}\mathrm{pH} \mathrm{4.5,} \\
\mathrm{H}_{3} \mathrm{PO}_{4}\end{array}$ & 5000 & 310 & $\begin{array}{l}\text { Obtain high power, } \\
\text { high exposure be- } \\
\text { herlor of tube and } \\
\text { tube elements. Test } \\
\text { terminated by lailure } \\
\text { Fallure attributed to } \\
\text { high jacket tempera- } \\
\text { tures Induced by heary } \\
\text { condilm build-up } \\
\text { causing severe plttind } \\
\text { corrosion of jackets. }\end{array}$ & $\begin{array}{l}64,69, \\
70,71 \\
72,73\end{array}$ \\
\hline $288 \mathrm{~A}$ & 30 oct 59 & 3 & 10 & $\begin{array}{l}7 \mathrm{TRC}, .020 \\
\text { Hat., } \mathrm{HH}\end{array}$ & 91 & $.29 \times 106$ & 270 & 310 & $\begin{array}{l}\text { Ph 19, } \\
\text { L1OH }\end{array}$ & 2000 & $\cdots$ & $\begin{array}{l}\text { Evaluate hot-headed } \\
\text { closure on rods. } \\
\text { Test was st1ll rum- } \\
\text { n1ng at end of Cy-59 } \\
\text { and had attained an } \\
\text { exposure of } 885 \mathrm{wND} / \mathrm{T}\end{array}$ & 74,75 \\
\hline
\end{tabular}




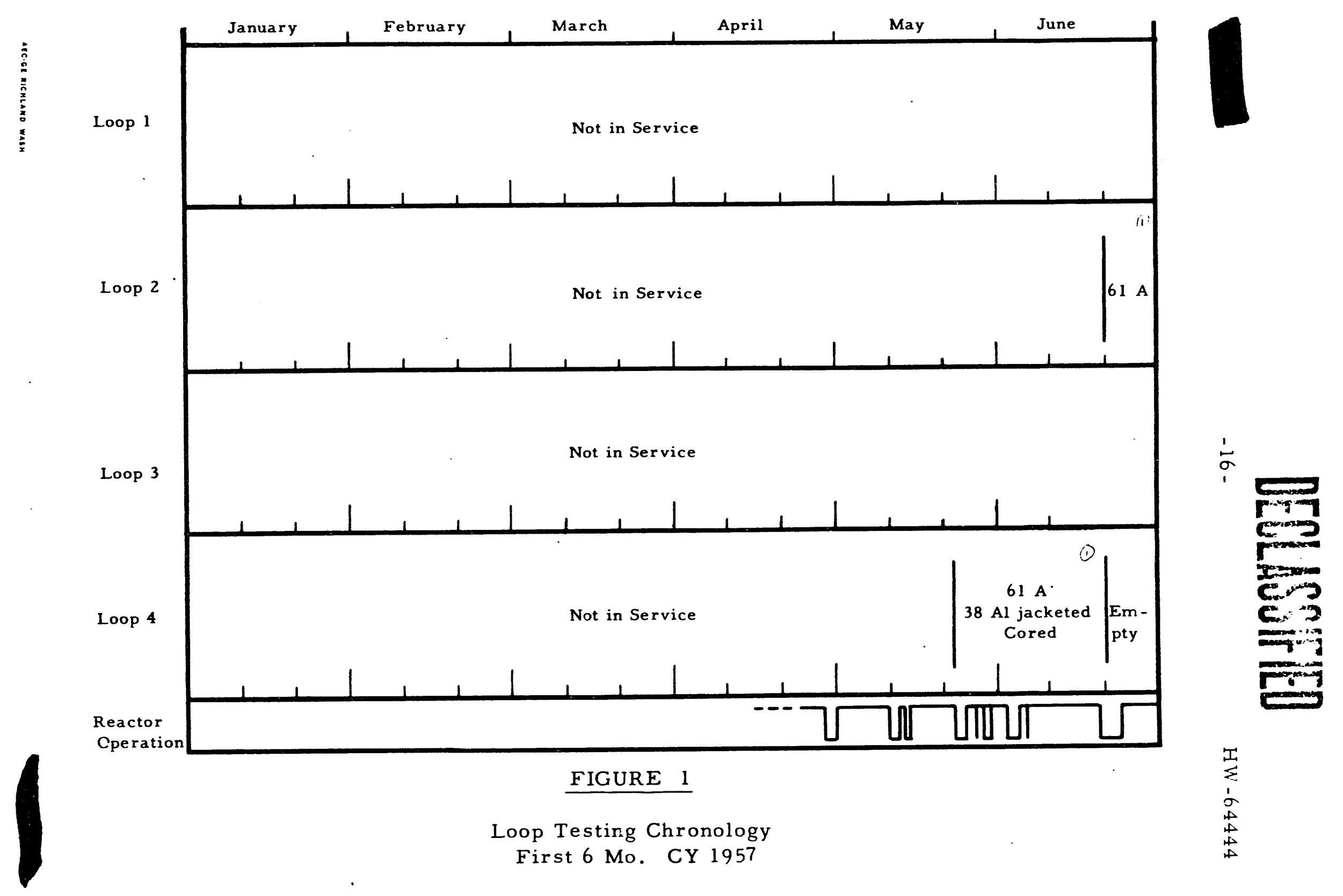




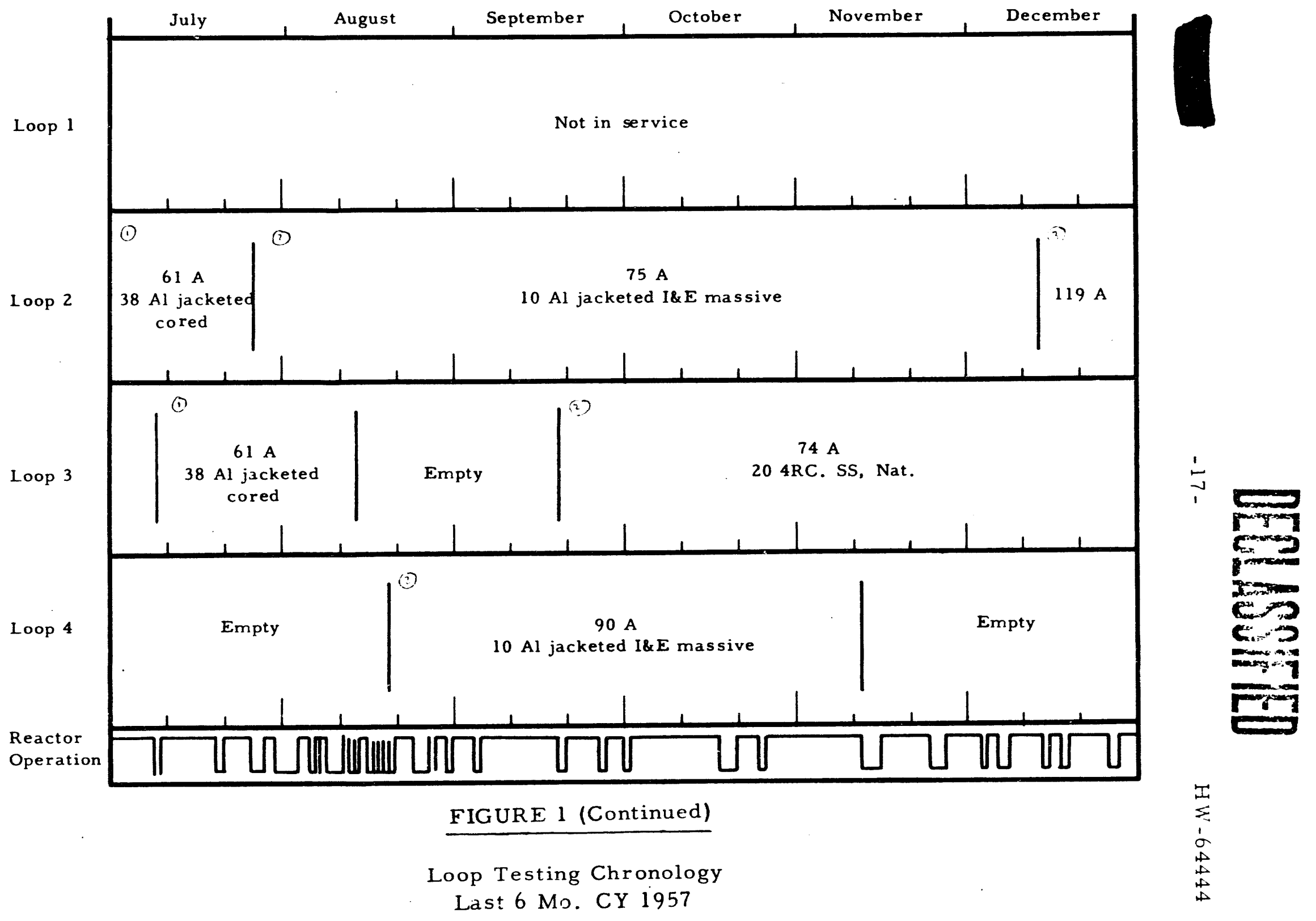




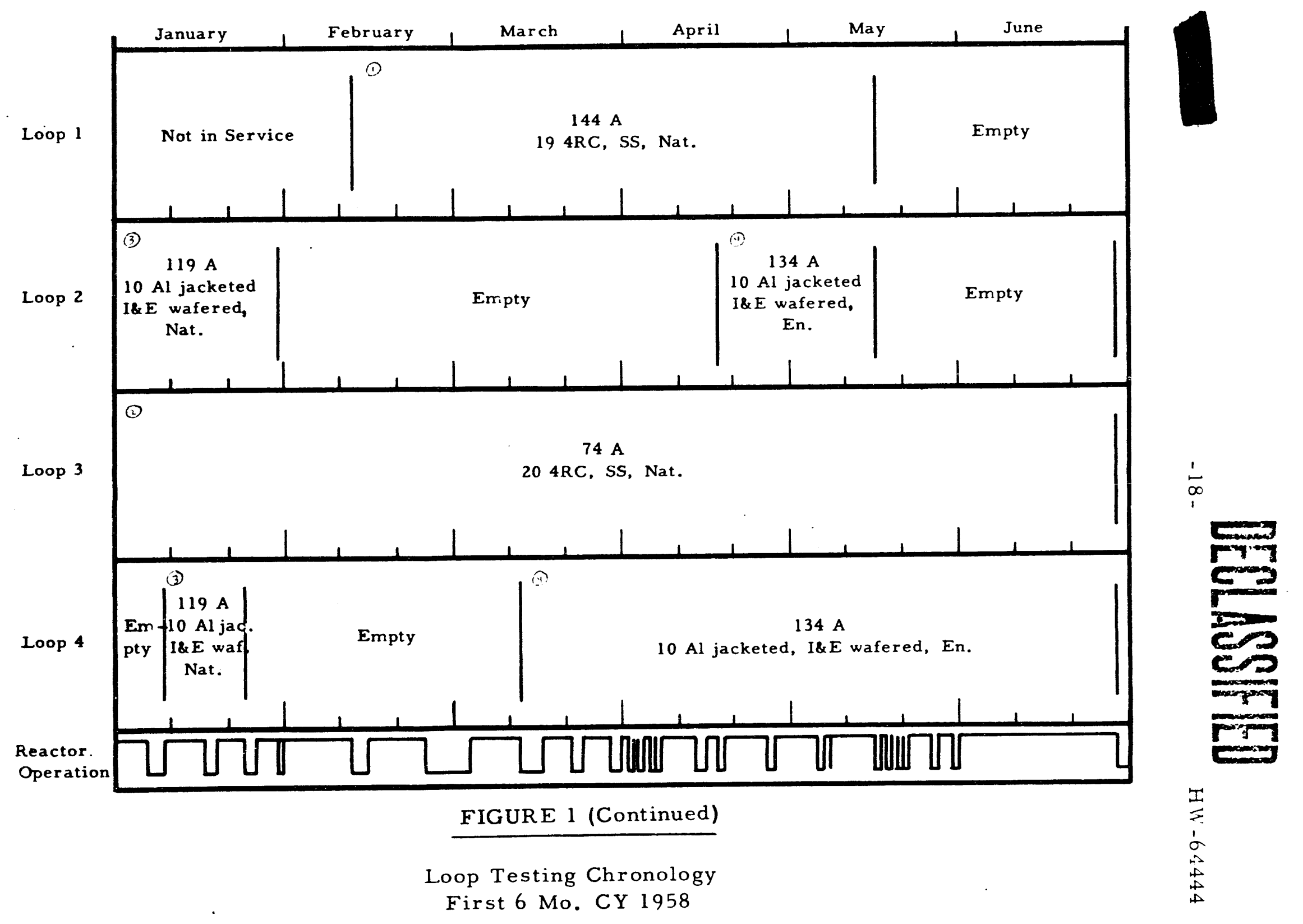




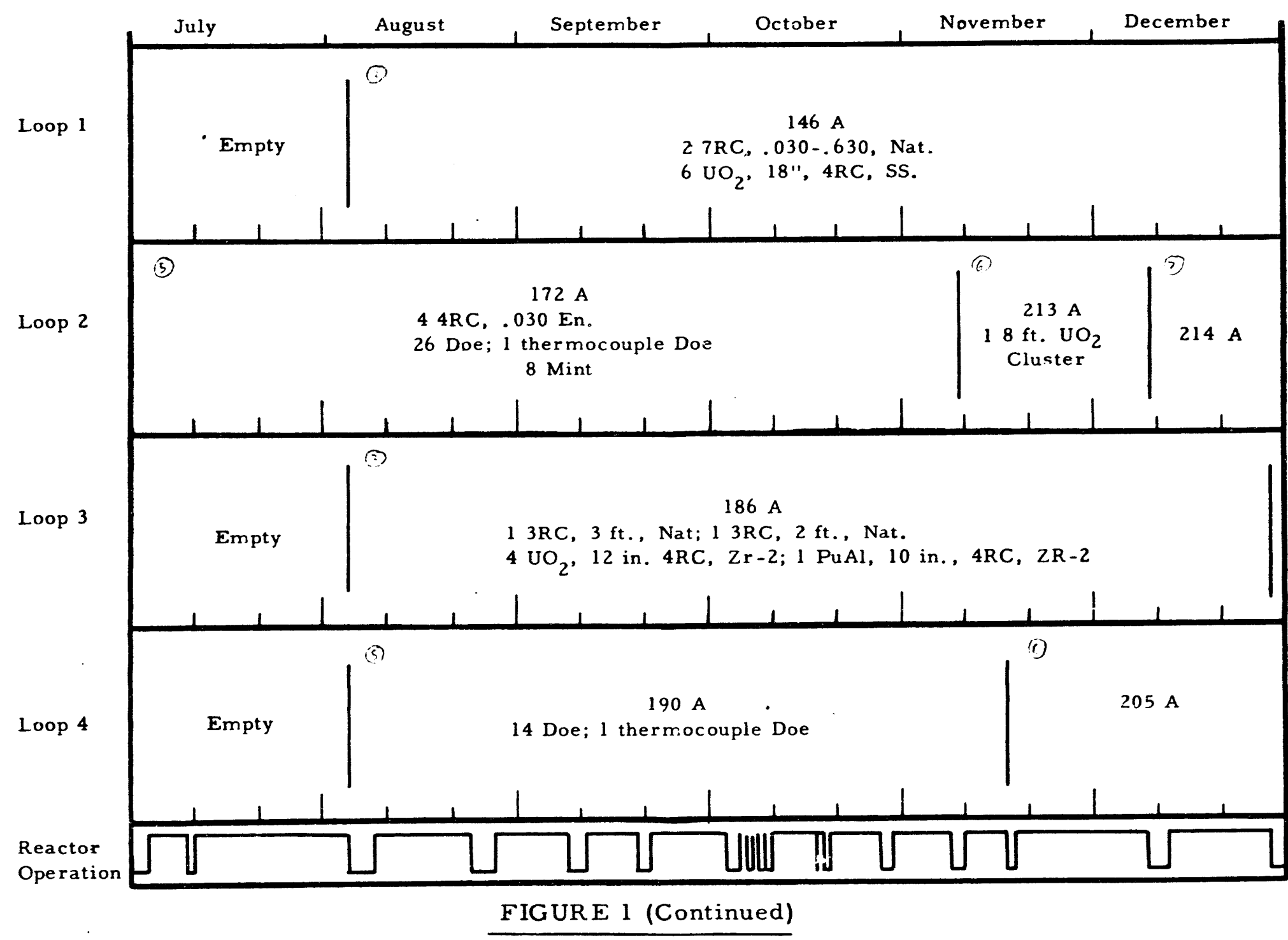

Loop Testing Chronology

Last 6 Mo. CY 1958 


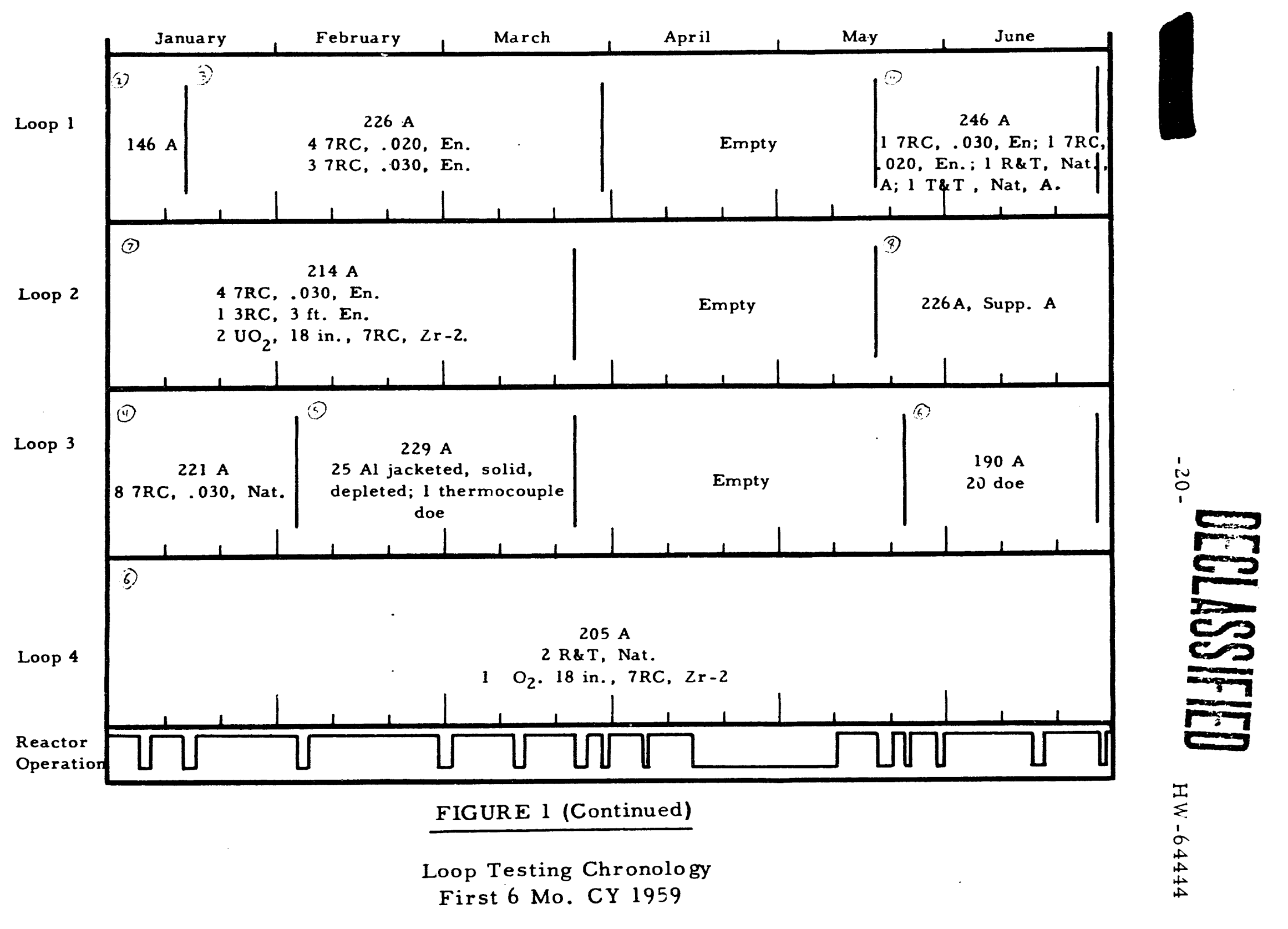




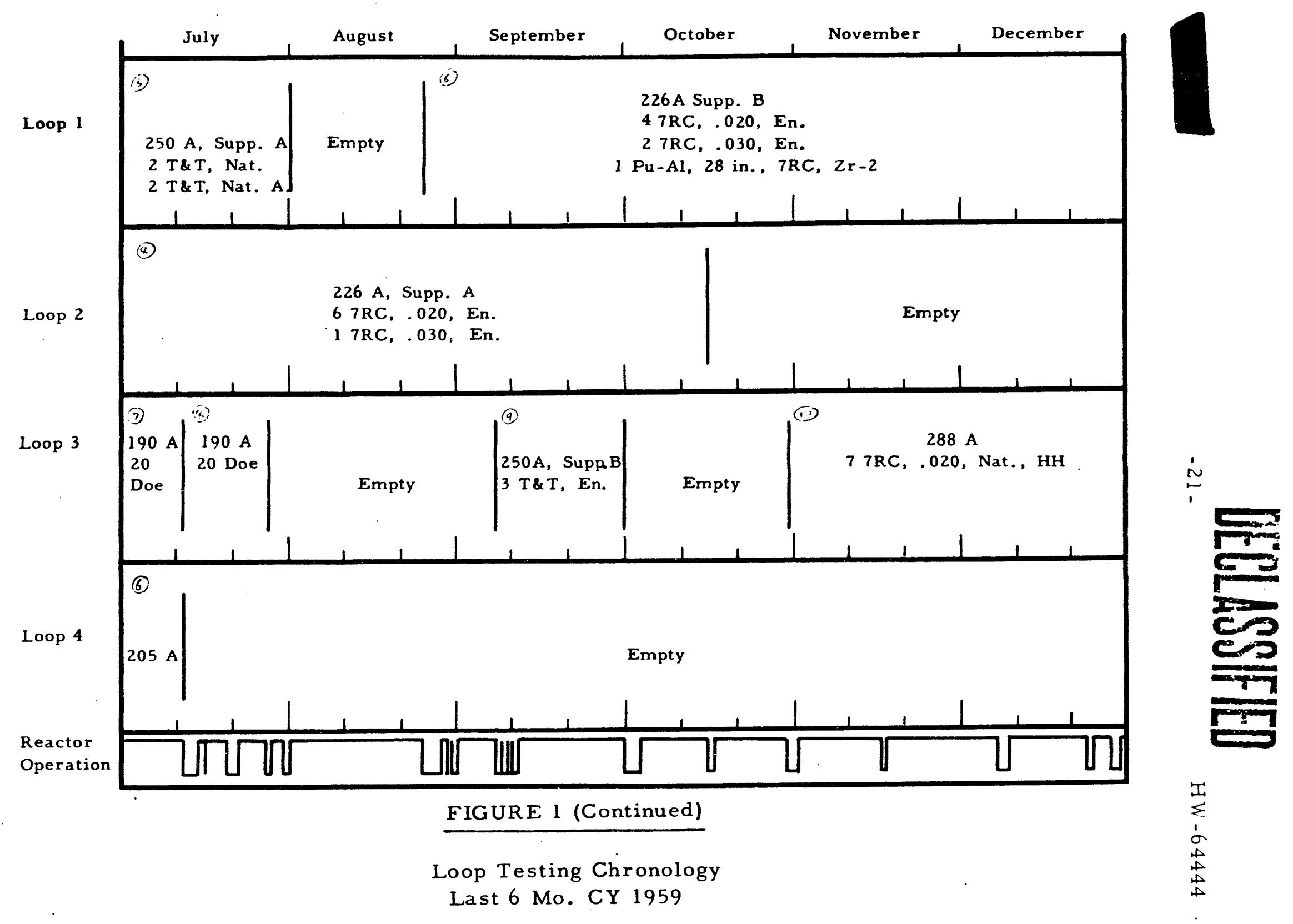




\section{DECLLSSFFIFD}

BIBLIOGRAPHY

1. Dulin, R.V. 1706 KER Coolant Test Facility Operations Manual, HW-45000 Rerised. January 23, 1958.

2. Process Technology Operat1on, IPD. Process Standards-Reactor, HW-46000-K. (SECRET)

3. Miller, M.R. Production Test IP-61-A, Irradidat1on of 1.44 Inch Diameter Cored Self-Supporting Flements in the KRR Fac1lity, BW-49023. March 26, 1957. (SECRET)

4. Kratzer, W.K. Production Test IP-75-A, Irradiation of 1.800 Inch Massive I\&E Fuel Flements in the 1706 KaR Recirculation Test Fac1lity, Loop K-2, Tube 2864 KI, HW-50623. July 15, 1957. (SECRET)

5. Kratzer, W.K. Production Test IP-90-A, Irradiation of 1.800 Inch Massive I\&a Fuel Flements in the $1706 \mathrm{KaR}$ Recirculation Test Fac1lity Loop K-4, Tube $4268 \mathrm{KK}, \mathrm{BW}-50773$. July 16, 1957. (SECRET)

6. Kratzer, W.K. Production Test IP-74-A, Irradiat1on of Stainless Steel Jacketed Four-Rod Cluster Fuel Elements in the 1706-KkR Recircualtion Test Fac1lity, Loop K-3, Tube $3565 \mathrm{KE}$, HW-51366. July 17, 1957. (SECRRT)

7. Kratizer, W.K. Supplement A to Production Test IP-74-A, Irradiation of Stainless Steel Jacketed Four-Rod Cluster Fuel Flements in the $1706-K R R$ Resirculation Test Fac1lity, Loop K-3, Tube 3565, HW-53385. october 29, 1957. (SECRFT)

8. Kratzer, W.K. Supplement B to Production Tegt IP-74-A, Irraaiation of Stainless Steel Jasketed Four-Rod Cluster Fuel Elements in the 1706-KER Recirsulation Tube Fac1lity, Loop K-3, Tube $3565 \mathrm{KE}$, HW-54195. December 16, 1957. (SBCRET)

9. Brandt, R.I. Radiometallurgical Examination of a Stainless Steel Jacketed Four-Rod Cluster Fuel Flement Irradiated under PT-IP-74-A, RK-246, BW-62880. December 1, 1959. (CONFIDENTIAL)

10. Lofftus, F.H. and Wheeler, K.R. Pre-Irradiation Testing of Lead-Dip Canned KER I\&E Wafer Type Fuel Blements, EW-52873. October 1, 1957. (COIF IDENTIAL)

11. Miller, M.R. and Kratzer, W.K. Production Test IP-119-A, Irradiation of Aluminum Jacketed Wafered Uranium Fuel Elements in the KaR Test Facility, HW-53226. Horember 19, 1957. (SECRFT)

12. Kratzer, W.K. Production Test IP-144-A Irradiation of Stainless Steel Jacketed Four-Rod Cluster Elements in KRR Loop 1, Tube $2160 \mathrm{KE}$, EW-54847. February 4, 1958. (SECRET)

13. Geering, G.T. KRR K-1-2 Fuel, HW-54936. February 12, 1958. (SFCRET) 
14. Kratzer, W.K. Outline of an Irradiation Test of $1.6 \%$ Enriched Hot Pressed Wafered Fuel Elements in the KER Loops, HW-54501. January 13, 1958. (SECRET)

15. Kratzer, W.K. and Killer, N.R. Production Test IP-134-A, Irradiation of $1.6 \%$ Fnriched Hot-Pressed Wafered Uranium Fuel Elements in the KER Reclrculation Test Fac1lity, HW-54274. February 27, 1958. (SECRET)

16. Kratzer, W.K. and M1ller, M.R. Supplement A to Production Tcst IP-134-A, Irradiation of $1.6 \%$ Enriched Hot-Pressed Wafered Uranium Fuel Elements In the KRR Rec1rculation Test Fac1l1ty, HW-55663. April 9, 1958. (SECRET)

17. Hayden, K.D. Hot-Press Elephant Slug Ruptures, HW-54686. January 27, 1958. (COAFIDWIAL)

18. Smith, E.A. Pre-Irradiat1on Tests Performed on Material for PT-IP-134-A, (KER Washer Element.s), BW-55034. Karch 5, 1958. (CONFIDENTIAL)

19. Doman, D.R. and Lobsinger, R.J. Interim Report No. 1: High Temperature Out-of-Reartor Evaluation of KER Fuel Elements. May 15, 1957 to Maroh 15, 1958., EW-55639. Apr11 15, 1958.

20. Merckx, K.R. Mechanical Conditions in Wafer Fuel Elements, HW-53312. October 22, 1957. (CONFIDENTIAL)

21. Kratzer, W.K. and Miller M.R. Production Test IP-172-A, Short Term, High Temperature Irradiation of Enriched Cluster Elements and $\mathrm{K}-388$ Iacketed Doe Elements in KaR Loops, HW-56297. June 10, 1958. (SECRET)

22. Kratzer, W.K. and Killer, M.R. Supplement A to Produstion Test IP-172-A, Short Term, High Temperature Irradiation of Enriched Cluster Elements and $\mathbf{M}=388$ Jacketed Foe Elemente in KRR Loops, HW-56996. August 4, 1958. (SECRET)

23. Claudson, T.T. Final Report: Irradiation Performance of Caextruded Enriched Uranium Fuel Rod PT-IP-172-A, HW-60520. May 26, 1959. (CONFIDENTIAL)

24. Doman, D.R. Thermosouple Slug Test Proposal for the $1706 \mathrm{KER}$ Test Fac1l1ty, HW-56286. June 6, 1958.

25. Kratzer, W.K. Produstion Test IP-146-A, Irradiation of Coextruded Bonded Zirealoy- $\hat{c}$ Jasketed Seven-Rod Sluster Fuel Elements in the KRR Recirculation Test Fasility, HW-55813. April 21, 1958. (SECRET)

26. Kratzer, W.K. Supplement A to Production Test IP-146-A, Irradiation of Coextruded Bonded Z1rcaloy-2 Jacketed Sever-Rod Cluster Fuel Flements in the KRR Recirculation Test Fac1lity, BW-55814. Apr11 21, 1958. (SECRET)

27. Gruber, W.J. Post-Irradiation Examination of KER 1-3 Seven-Rod Cluster Fuel Elements (RM-277), HW-62426. October 19, 1959. 


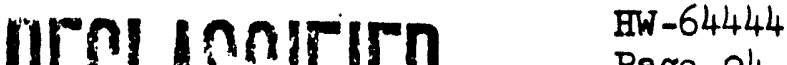 \\ Page 24}

28. Kratzer, W.K. and Mller, M.R. PT-IP-186-A, High Temperature Irradiat1on of Two and Three Foot Long Cluster Flements in the KRR Loops, HW-56549. July 9, 1958. (SFCRET)

29. Bates, J. I., Millhollen, K.K. and Stenquist, D.R. Four Rod Cluster Fuel Flements for Irradiation in KRR-3, HW-56693-RD. July 9, 1958.

30. Bally, W.J. Proposal for the Irradiation Testing of Al-Pu Alloy Fuel Element Clusters, HW-53511. Tovember 7, 1957. (SECRET)

31. Hall, R.B. Failure of Al-Pu Alloy Fuel Elements in a KFR Loop, HW-54210. December 16, 1957. (SRCRET)

32. Miller, H.R. and Kratzer, W.K. Produetion Test IP-190-A, Irradiation of Aluminum Clad Doe Flements in the KRR Recirculation Test Facility, HK-56855. July 24, 1958. (SECRET)

33. Miller, N.R. and Kratzer, W.K. Supplement A, Production Test IP-190-A, Irradiation of Aluminum Clad Doe Elements in the KRR Test Fasility, HW-57614. September 29, 1958. (SECRET)

34. Dickinson, D.R. Corrosion Rate of Aluminum Clad Elements in KRR-3, HW-60207. April 29, 1959. (SFCRET)

35. Miller, N.R. and Kratzer, W.K. Supplement B - Production Test IP-190-A, Irradiation of Aluminum Clad Doe Elements in the KER Test Facility, EW-60244. May 1, 1959. (SECRET)

36. Sharp, F.E. KFR Loop 3 operating Report, Aluminum Testing In KER Loop 3 from May 23, 1959 to July 26, 1959, HW-61786. September 1, 1959. (SFCRkPT)

37. Teats, R. Radiometallurgieal Examination of Doe Element Failure Irradiated Under PT-IP-190-A (RM-402), HW-62953. December 4, 1959. (CONFIDENTIAL)

38. Ayres, J.A. Evaluation of Aluminum for Use in Reactors Cooled By High Temperature Reclrculating Water, HW-61757. September 2, 1959.

39. Kratzer, W.K. Production Test IP-213-A, Irradiation of an Eight Foot UO2 Seren-Rod Cluster Flement, HW-57865. October 22, 1958. (SFCRWT)

40. Kratzer, W.K. Operating Conditions for PT-IP-213-A, Irradiation of an Fight Foot Uु2 Seven-Rod Cluster Flement, HW-58104. November 6, 1958.

41. Kratzer, W.K. PT-IP-205-A, Irradiation of Z1rcaloy-2 Jacketed Rodand-Tube Flements in the KkR Loops, HW-57732, October 9, 1958. (SFCRFT)

42. Kratzer, W.K. Operating Conditions for PT-IP-205-A, Irradiations of Zircaloy-2 Jacketed Rod-and-Thbe klements in the KkR Loops, HW-58259. November 25, 1958。 (SFCRWT)

43. Swanson, W.I. KGR Loop 4 (4268) Operating Report, Test Number K 4-6 Production Test IP-205-A, Irradiation of Z1rcaloy-2 Jacketed Rod-and Tube-F'lements, FW-61783. September 1, 1959. (SECRET) 


\section{UECLLSPFIFD}

44. Gates, J.E. Post-Irradiation Examination of Zircaloy-2 Clad Uranium Fuel Rlements, GrH-24848. March 17, 1960. (CONFIDENTIAL-UNDOCUMENIED)

45. Claudson, T.T. Proposal for Irradiation of 1.6 Per Cent Enriched 7-Rod Cluster Fuel Flements, HW-57056. August 7, 1958. (SECRET)

46. Kratzer, H.K. Production Test IP-214-A, Irradiation of Enriched Zircaloy-2 Jacketed Seven-Rod Cluster klements, HW-58253. Hovember 24, 1958. (SECRET)

47. Kratzer, W.K. Supplement A to Production Test IP-214-A, Irradiation of Fnriched Zircaloy-2 Jacketed Seven-Rod Cluster Elements, HW-59700. March 19, 1959. (SECRET)

48. Small, T.A. KRR Loop 2 Operating Report, HW-61785. September 1, 1959. (SECRET)

49. Kratzer, W.K. Production Test IP-22l-A, Measurement of Flow Channel Temperatures in Seven-Rod Cluster Flementr in the KER Loops, HW-58438. December 8, 1958. (SECRET)

50. Kratzer, W.K. Supplement A to PT-IP-22l-A, Measurement of Flow Channel Temperatures in Seren-Rod Cluster Elements in the KER Loops, HW-58583. December 17, 1958. (SECRET)

51. Teats, R. Radiametallurgical Examination of PT-IP-22l-A, Measurement of Flow Channel Temperature in Seven-Rod Cluster Fuel Elemen: (RM-287), BW-60754. June 16, 1959. (CONFIDENTIAL)

52. Kra zer, W.K. Production Test IP-226-A, Irradiation of Enriched SevenRod Cluster Elements with I'wenty and Thirty Kil Zircaloy-2 Jackets, EW-58781. January 8, 1959. (SECRET)

53. Kratzer, W.K. Production Test IP-229-A, Braluation of the Uranium Al-S1 Bond at High Temperature, HW-58929.' January 19, 1959. (SECRET)

54. Sharp, F.E. KER Loop To. 3 Al-S1 Bond Test, HW-60421. Yay 18, 1959. (SECRET)

55. Goffard, J.W. and Wheeler, R.G. Progress Report, High Temperature High Pressure Bake Testinis and Diffusion Studies of Aluminum Clad Fuel Elements, HW-56300. July 25, 1958.

56. Doman, D.R. Proposed Design for Front Face Thermocouple Lead-Out for the KRR Loops, Hik-58829. January 12, 1959

57. Bokish, K.P. Ultrasonic Bond Testing of Irradiated Fuel Elements, HW-55028. Nay 9, 1958. (SFCRET)

58. Kratzer, W.K. Supplement A to Production Test IP-226-A, Irradiation of Enrlched Seren-Rod Cluster Elements with Twenty and Thirty Kil Zircaloy-2 Jackets, $\mathrm{HW}-60112$. Apr11 21, 1959. (SERCRET) 
59. Small, T.A. KRR Loop 2 0perating Report, Test No. K 2-8, PT-IP-226-A, HW-62910. December 1, 1959. (SECRET)

60. Kratzer, W.K. Production Test IP-246-A, Low Exposure Irradiation of Zircaloy-2 Jacketed Seren-Rod Cluster Flements and Zircaloy-2 Jacketed Zirconium Alloy Tube-and-Tube Flements, HW-59747. April 20, 1959. (SECRET)

61. Kratzer, W.K. Supplement A to Production Test IP-246-A, Low Exposure Irradiation of Z1rcaloy-2 Jacketed Seven-Rod Cluster Flements and Zircaloy-2 Jacketed Zirconium Alloy Tube-and-Tube Flements, HW-60271. May 5, 1959. (SFCRET)

62. Eikum, L.M. KRR Loop Ko. 1 - Low Exposure Irradiation of Zircaloy-2 Jacketed Seren-Rod Cluster Elements and Zircaloy-2 Jacketed Rod-and-Tube and Tube-and-Tube Elements, HW-61334. July 30, 1959

63. Gruber, W.J. Post Irradiation Examination of Seven-Rod Cluster Element with Yodified End Closures, PT-IP-246-A, (RN-552), HW-62612. December 1, 1959. (SECRET)

64. Kratzer, W.K. Production Test IP-250-A, Irradiation of Z1rcaloy-2 Jacketed Tube-and-Tube Elements in the KRR Loops, HW-60551. June 1, 1959. (SECRET)

65. Kratzer, W.K. Supplement A to Production Test IP-250-A, Irradiation of Zircaloy-2 Jacketed Tube-and-Tube Flements in the KER Loops, HW-60719. June 12, 1959. (SECRET)

66. Fikum, L.M. KRR Loop I Operating Report, A Report on the Irradiat ion of Z1rcaloy-2 Jacketed Tube-and-Thube Elements in KRR LoOp \#I, HW-61784. September 1, 1959. (SFCRET)

67. Kratzer, W.K. Supplement B to Production Test IP-226-A, Irradiat 1on of Fnriched Seven-Rod Cluster Flements with Twenty and Thirty Mil Zircaloy-2 Jackets, HW-61541. August 13, 1959. (SFCRWT)

68. Doman, D.R. Out-of-Reactor Tests on Pu-Al Type PRTR Flements for 1706 KTRR Testing, HW-61517. August 7, 1959.

69. Kratzer, W.K. Supplement B to Production Test IP-250-A, Irradiation of Zircaloy-2 Jacketed Tube-and-Tube Flements in the KaR Loops, HW-61160. July 20, 1959. (SECRET)

70. Kratzer, W.K. Revised Operating Conditions for Enriched Tube-and-Tube Blements in the KER Loops Under PT-IP-250-A, Supplement B, HW-62059. September 24, 1959. (SFCRET)

71. Sharp, F.F. KRR Loop 3 Operating Report, Tube-in-Tube Flement Test, HW-62909. December 1, 1959. (SECRWT)

72. Last, G.A. and Geering, G.T. Examination of Ruptured KRR Tube-inTube Fuel klement from IP-250-A, Supplement B, HW-62428. October 19, 1959. (SECRET) 


\section{DENISSEFED

73. Perrigo, L.D. Radiochemistry for the Rupture of a Tube-in-Tube Fuel Element in KRR Loop 3, HW-62677. November 5, 1959. (SECRET)

74. Allison, G.S. KER Seven-Rod Cluster Fuel Element Charge. August 17, 1959 (COKFIDENTIAL-UTDOCUNANTED)

75. Kratzer, W.K. Production Test IP-288-A, Evaluation of Seren-Rod Cluster Elements with Modified End Closures, HW-62416. October 16, 1959. (SECRET)

76. Wheeler, R.G. Seren-Rod Cluster Irradation of Fuel Rods with End Closure and Fnd Fixture, HW-63715. February 1, 1960. (SECRET)

77. Perrigo, L.D. Radiochemistry for the Rupture of a Z1rcaloy-2 Clad Seven-Rod Cluster Fuel Element in KRR Loop 2, HW-63599. January 22, 1960. (SECRET)

78. Christensen, J.A. Irradiation Growth of Uranium-Zirconium Alloy, Tubular Fuel Elements, HW-61636. August 24, 1959. (SECRET) 

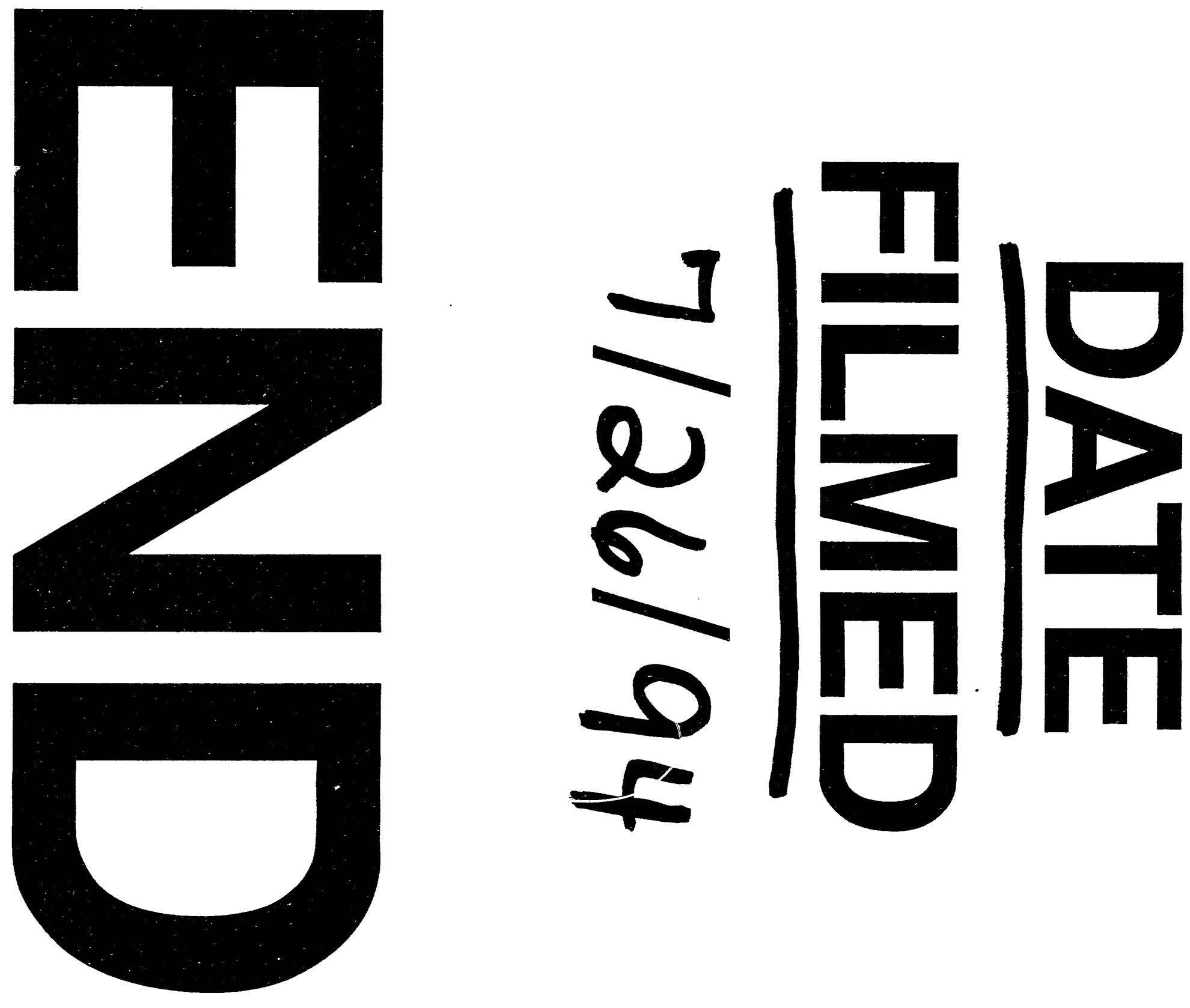
